IASSNS-HEP-92/62

RU-92/37

\title{
Free Field Representation for the Classical Limit of Quantum Affine Algebra
}

\author{
Sergei Lukyanov $\dagger^{*}$ \\ Department of Physics and Astronomy \\ Rutgers University,Piscataway, NJ 08855-049 \\ Samson L. Shatashvili $\ddagger \#$ \\ School of Natural Science \\ Institute for Advanced Study \\ Olden Lane \\ Princeton, NJ 08540
}

Free field representation for the classical limit of quantum affine algebra is constructed by simple deformation of the known expressions from WZW theory.

September, 92

$\dagger$ Research supported by grant DE-FG05-90ER40559

* On leave of absence from L.D.Landau Institute for Theoretical Physics, Kosygina 2, Moscow, Russia.

$\ddagger$ Research supported by DOE grant DE-FG02-90ER40542.

\# On leave of absence from St. Petersburg Branch of Mathematical Institute (LOMI), Fontanka 27, St.Petersburg 191011, Russia. 
The progress achieved during the last decade in CFT is a result of a successful applications of the representation theory of infinite dimensional algebras to physics problems [1], [2], [3]. In the class of CFT where one can speak about classical theory, representation theory arises via geometric quantization of classical phase space. For compact WZW model this is probably best understood and it turned out that classical phase space for chiral part of the theory can be viewed as the space of mappings of the segment to the group [4], [5], [6], [7], [8]. In this language the group element becomes the simplest chiral vertex operator after quantization. The existence of Darboux variables on phase space leads to free field representation for vertex operators and symmetry algebra itself [9], [10] and this could be viewed as classical explanation of Feigin-Fuchs-Dotsenko-Fateev integral representation for conformal blocks. The latter practically is the most powerful technical way of constructing the correlation functions in Rational CFT.

There are serious indications in the favor of existence of the analogous structure in the massive integrable theories (for latest developments see [11], [12], [13], [14], [15]). F. Smirnov [11] have pointed out that the equations for form-factors in the $S U(2)$ Thirring model [16], [17] coincides with the quantum Knizhnik-Zamolodchikov equation. I. Frenkel and N. Reshetikhin had investigated in details the mathematical aspects of quantum Knizhnik-Zamolodchikov equation and it's relation to Smirnov's type of form-factors. Moreover, in the recent papers from Kyoto school [13], [14] [15], the anti-ferroelectric XXZHamiltonian was diagonalized in the thermodynamic limit using the representation theory of quantum affine algebra $U_{q}(\widehat{s l(2)})$.

In this paper, we address the question that naturally emerges from the above discussion: could integrable massive theories be formulated in a similar fashion as the CFT? This question seems very complicated, so we should first consider the classical case.

1. Let us recall a few facts about classical phase space of chiral WZW model [4], [5], [6], [7], [8] . Consider the group of mappings of interval $[0,2 \pi]$ into the finite dimensional Lie group $S L(2)$ with elements $g(\sigma), \sigma \in[0,2 \pi]$. The algebra of functions on this manifold has natural Poisson structure, which can be described in the following way. $\mathrm{We}$ denote the Cartan-Weyl basis of generators of the Lie algebra $s l(2)$ as $E_{+}, H, E_{-}$. Then the Poisson brackets for matrix elements $g(\sigma)$ have the form

$$
\left\{g(\sigma) \otimes, g\left(\sigma^{\prime}\right)\right\}=\left(r_{+} \Theta\left(\sigma-\sigma^{\prime}\right)+r_{-} \Theta\left(\sigma^{\prime}-\sigma\right)\right) g(\sigma) \otimes g\left(\sigma^{\prime}\right)
$$

1 We prefare to speak about Poisson structure instead of symplectic one. 
where

$$
r_{ \pm}= \pm h\left(\frac{H \otimes H}{2}+E_{ \pm} \otimes E_{\mp}\right)
$$

and

$$
\Theta(\sigma)= \begin{cases}1, & \sigma>0 \\ 0, & \sigma<0\end{cases}
$$

This structure induces linear bracket for currents $J(\sigma)=-\frac{2 \pi}{h} g^{-1}(\sigma) \partial_{\sigma} g(\sigma)$ :

$$
\frac{1}{2 \pi}\left\{J^{a}(\sigma), J^{b}\left(\sigma^{\prime}\right)\right\}=f^{a b c} J^{c}(\sigma) \delta\left(\sigma-\sigma^{\prime}\right)-\frac{2 \pi}{h} \delta^{a b} \delta^{\prime}\left(\sigma-\sigma^{\prime}\right) .
$$

In CFT the natural boundary conditions for currents are periodical ones. So one can decompose the currents in Fourier series

$$
J^{a}(\sigma)=\sum_{-\infty}^{+\infty} J_{-n}^{a} e^{i n \sigma},
$$

then the commutation relations for generators $J_{n}^{a}$ will have the form

$$
\left\{J_{n}^{a}, J_{m}^{b}\right\}=f^{a b c} J_{n+m}^{c}+\frac{2 i \pi}{h} n \delta^{a b} \delta_{n,-m} .
$$

After quantization relation (11) transforms to

$$
g(\sigma) \otimes g\left(\sigma^{\prime}\right)=g\left(\sigma^{\prime}\right) \otimes g(\sigma) R, \quad \sigma>\sigma^{\prime}
$$

where $R$ is the universal quantum $R$-matrix for $U_{q}(\operatorname{sl}(2))$ [18] and (4) becomes the commutation relations for affine Lie algebra. Let us note that the matrix elements of quantum field $g(\sigma)$ are the vertex operators. It is known that the Darboux variables for Poisson structure (1) are classical analog of Wakimoto free fields. More precisely, in the Gauss parameterization

$$
\begin{gathered}
g(\sigma)=\left(\begin{array}{ll}
1 & 0 \\
\psi & 1
\end{array}\right)\left(\begin{array}{cc}
e^{\phi / \sqrt{2}} & 0 \\
0 & e^{-\phi / \sqrt{2}}
\end{array}\right)\left(\begin{array}{ll}
1 & \gamma \\
0 & 1
\end{array}\right) \\
\psi(\sigma)=\int_{\sigma}^{2 \pi} d \mu \beta(\mu) e^{-\sqrt{2} \phi(\mu)}
\end{gathered}
$$

the Poisson structure (11) is canonical

$$
\begin{aligned}
& \left\{\phi(\sigma), \phi\left(\sigma^{\prime}\right)\right\}=\frac{h}{2} \epsilon\left(\sigma-\sigma^{\prime}\right) \\
& \left\{\gamma(\sigma), \beta\left(\sigma^{\prime}\right)\right\}=h \delta\left(\sigma-\sigma^{\prime}\right) \\
& \left\{\phi(\sigma), \gamma\left(\sigma^{\prime}\right)\right\}=\left\{\phi(\sigma), \beta\left(\sigma^{\prime}\right)\right\}= \\
& =\left\{\gamma(\sigma), \gamma\left(\sigma^{\prime}\right)\right\}=\left\{\beta(\sigma), \beta\left(\sigma^{\prime}\right)\right\}=0
\end{aligned}
$$


where $\epsilon(\sigma)=\Theta(\sigma)-\Theta(-\sigma)$. One can check that the function $\int_{0}^{2 \pi} d \mu \beta(\mu) e^{-\sqrt{2} \phi}$ commutes with whole current algebra; this object after quantization is the screening operator in Feigin-Fuchs-Dotsenko-Fateev integral representation for correlation functions.

This is the classical picture which is the underline of the present technique in CFT; although the main technical results could be obtained in different fashion (and in many cases they were) we think this interpretation deserves attention.

2 . In this letter the natural, from our point of view, generalization of the picture described above, will be suggested. This construction is based on Darboux variables emerging from Gauss decomposition.

Let us consider the group with elements being the mappings $Z(\theta) \in S L(2)$. Keeping in mind the applications of this construction for massive integrable models of field theory [11], [12], [13], [14], [15], we will consider the parameter $\theta$ as the rapidity, so the whole line is a natural range of its variation. One should note this important difference with the conformal case. The group element $Z(\theta)$, as $g(\sigma)$, admits the Gauss decomposition (6). We will preserve the notation (6) for the components of $Z(\theta)$, but will consider a more general form for function $\psi$ :

$$
\begin{aligned}
& Z(\theta)=\left(\begin{array}{cc}
e^{\phi / \sqrt{2}} & \gamma e^{\phi / \sqrt{2}} \\
\psi e^{\phi / \sqrt{2}} & e^{-\phi / \sqrt{2}}+e^{\phi / \sqrt{2}} \gamma \psi
\end{array}\right), \\
& \psi(\theta)=\int_{-\infty}^{+\infty} d \theta^{\prime} \beta\left(\theta^{\prime}\right) e^{-\sqrt{2} \phi\left(\theta^{\prime}\right)} h\left(\theta^{\prime}-\theta\right),
\end{aligned}
$$

where kernel $h(\theta)$ is a numerical function. Moreover, we admit more general form for Poisson structure for $\phi$, keeping others $\beta, \gamma$ the same as in (7):

$$
\begin{aligned}
& \left\{\phi(\theta), \phi\left(\theta^{\prime}\right)\right\}=\frac{h}{2} \rho\left(\theta-\theta^{\prime}\right), \\
& \left\{\gamma(\theta), \beta\left(\theta^{\prime}\right)\right\}=h \delta\left(\theta-\theta^{\prime}\right), \\
& \left\{\phi(\theta), \gamma\left(\theta^{\prime}\right)\right\}=\left\{\phi(\theta), \beta\left(\theta^{\prime}\right)\right\}= \\
& =\left\{\gamma(\theta), \gamma\left(\theta^{\prime}\right)\right\}=\left\{\beta(\theta), \beta\left(\theta^{\prime}\right)\right\}=0,
\end{aligned}
$$

here $\rho(\theta)$ should be an odd function

$$
\rho(\theta)=-\rho(-\theta)
$$

From the requirement that matrix elements of $Z(\theta)$ form quadratic Poisson algebra under (9) we immediately obtain two functional equations: 


$$
\begin{gathered}
\rho(\theta) h\left(\theta^{\prime}\right)=2 h\left(\theta^{\prime}-\theta\right) h(\theta)-\rho\left(\theta^{\prime}-\theta\right) h\left(\theta^{\prime}\right), \\
\rho\left(\mu-\mu^{\prime}\right)\left[h(\mu-\theta) h\left(\mu^{\prime}-\theta^{\prime}\right)-h\left(\mu-\theta^{\prime}\right) h\left(\mu^{\prime}-\theta\right)\right]= \\
=\rho\left(\theta-\theta^{\prime}\right)\left[h(\mu-\theta) h\left(\mu^{\prime}-\theta^{\prime}\right)+h\left(\mu^{\prime}-\theta\right) h\left(\mu-\theta^{\prime}\right)\right]- \\
-2 h\left(\theta-\theta^{\prime}\right) h(\mu-\theta) h\left(\mu^{\prime}-\theta\right)+2 h\left(\theta^{\prime}-\theta\right) h\left(\mu-\theta^{\prime}\right) h\left(\mu^{\prime}-\theta^{\prime}\right) .
\end{gathered}
$$

At the same time one could check that the Poisson algebra for matrix $Z(\theta)$ should have the form :

$$
\left\{Z(\theta) \otimes, Z\left(\theta^{\prime}\right)\right\}=r\left(\theta-\theta^{\prime}\right) Z(\theta) \otimes Z\left(\theta^{\prime}\right)
$$

where

$$
r(\theta)=h\left[\frac{\rho(\theta)}{2} H \otimes H+h(\theta) E_{+} \otimes E_{-}-h(-\theta) E_{-} \otimes E_{+}\right]
$$

One must note that the first equation (11), is nothing more but classical Yang-Baxter equation for $r$-matrix (14), which is equivalent to Jacobi identity for Poisson structure (13). The nature of the second identity (12), seems to be less clear.

The systems of functional equations (11), (12) admits three type of solutions 2 :

i. constant solution

$$
\rho(\theta)=\epsilon(\theta), h(\theta)=\Theta(\theta)
$$

ii. rational solution

$$
\rho(\theta)=2 h(\theta)=V \cdot P \cdot \frac{1}{\theta}
$$

iii. trigonometric solution

$$
\begin{aligned}
& \rho(\theta)=V \cdot P \cdot \operatorname{coth}(\kappa \theta) \\
& h(\theta)=V \cdot P \cdot \frac{1}{1-\exp (-2 \kappa \theta)}
\end{aligned}
$$

where $\kappa$ is an arbitrary parameter and we denote by V.P. the principal value.

All of this solution are well known in theory of classical Yang-Baxter equation [19]. The trigonometric solution is the most general one; the constant and rational solutions could be obtained from it in the limits $\kappa \longrightarrow+\infty$ and $h, \kappa \longrightarrow 0 ; \frac{h}{\kappa} \longrightarrow$ const respectively. Also, for the limit $\kappa \longrightarrow \infty Z(\theta)$ obeys the same algebra as $g(\sigma), \psi$ from (8) has the

2 up to equivalence which in terms of $r(\theta)$ translates as

$$
r\left(\theta-\theta^{\prime}\right) \longrightarrow \Omega(\theta) \otimes \Omega\left(\theta^{\prime}\right) r\left(\theta-\theta^{\prime}\right) \Omega(\theta)^{-1} \otimes \Omega\left(\theta^{\prime}\right)^{-1}, \Omega(\theta)=\exp (\xi H \theta)
$$

where $\xi$ is constant number. 
form (6) and the Poisson structure (9) tends to (7). Thus, bellow we'll consider only trigonometric solution.

We will define the currents in the spirit of the work [11]. In fact two type of currents could be defined:

$$
\begin{gathered}
L(\theta)=Z\left(\theta+\frac{1}{\kappa}\right) Z^{-1}(\theta), \\
L^{\prime}(\theta)=Z^{-1}(\theta) Z\left(\theta+\frac{1}{\kappa}\right) .
\end{gathered}
$$

It is simple to check that in the limit $\kappa \longrightarrow+\infty$ the currents $L^{\prime}(\theta)$ can be written in the form $L^{\prime}(\theta)=1-\frac{2 \pi}{h \kappa} J(\theta)+O\left(\frac{1}{\kappa^{2}}\right)$, where

$$
\begin{aligned}
& -\frac{2 \pi}{h} J_{3}=\partial_{\theta} \phi+\sqrt{2} \beta \gamma, \\
& -\frac{2 \pi}{h} J_{-}=-\beta, \\
& -\frac{2 \pi}{h} J_{+}=\beta \gamma^{2}+\sqrt{2} \gamma \partial_{\theta} \phi+\partial_{\theta} \gamma
\end{aligned}
$$

and the Poisson brackets for variables $\gamma, \beta, \phi$ are defined by (7). One can recognize in (20) the classical limit of the Wakimoto representation for currents, so currents in (20) satisfy the Poisson algebra (3).

The currents $L(\theta)$ from (18) form the quadratic algebra :

$$
\begin{aligned}
& \left\{L(\theta) \otimes L\left(\theta^{\prime}\right)\right\}=r\left(\theta-\theta^{\prime}\right) L(\theta) \otimes L\left(\theta^{\prime}\right)+L(\theta) \otimes L\left(\theta^{\prime}\right) r\left(\theta-\theta^{\prime}\right) \\
& -L(\theta) \otimes 1 r\left(\theta-\theta^{\prime}-\frac{1}{\kappa}\right) 1 \otimes L\left(\theta^{\prime}\right)-1 \otimes L\left(\theta^{\prime}\right) r\left(\theta-\theta^{\prime}+\frac{1}{\kappa}\right) L(\theta) \otimes 1,
\end{aligned}
$$

the latter is the algebra defined by Reshetikhin-Semenov-Tian-Shansky [20]. They had shown in [20] that (21) is the classical limit of quantum affine algebra $U_{q}(\widehat{s l(2)})$ [18]. The limiting procedure $\kappa \longrightarrow \infty$, when $Z \longrightarrow g$ differs from one described in [20] for $L(\theta)$. The procedure of [20] requires the replacement $\kappa \longrightarrow i \kappa$ in (18) and (21). We must note that there are difficulties in this analytical continuation of our expression for $Z(\theta)$, because of the presence of principal value in its definition ( see (8),(9),(17)).

Allowed boundary conditions for the fields $L(\theta)$, as well as natural expansion for them, are those that are consistent with the kernel (17) and Poisson structure (9). From the other side what are natural boundary conditions from the point of view of massive Field Theory for $Z(\theta)$ and $L(\theta)$ is not clear at all. It seems to be related to the question of analytical properties, mentioned above. This is important to understand for further progress. We hope that the free field representation may help to make it clear. 
At the end, let us recall that the quantum operators $Z(\theta)$ in massive integrable theories have a meaning of creation operators for asymptotic states, i.e. some kind of FaddeevZamolodchikov operators. One would like to have the proper quantum analog of the free field representation for $Z(\theta)$ which will allow to construct integral representation for correlation functions in integrable models.

Note added. When this work was finished we received the papers [21], [22], where the free field representation of $U_{q}(\widehat{s l(2)})$ in different approach was developed. It would be interesting to understand the relation of these two pictures that looks very different at this moment.

Acknowledgment: We are grateful to L. D. Faddeev, V. A. Fateev, I. B. Frenkel, N. Yu. Reshetikhin and A. B. Zamolodchikov for useful discussions. Part of this work was done during the visit of one of us (S.Sh) to String Theory Group at Rutgers; he thanks the members of this group for warm hospitality. 


\section{References}

[1] A. A. Belavin, A. M. Polyakov and A. B. Zamolodchikov, Nucl.Phys. B241 (1984) 333.

[2] E. Witten, Commun. Math. Phys. 92 (1984) 455.

[3] V. G. Knizhnik and A. B. Zamolodchikov, Nucl. Phys. B247 (1984) 83.

[4] A. Alekseev and S. Shatashvili, Commun. Math. Phys. 133 (1990) 353.

[5] L. D. Faddeev, Comm.Math.Phys., 132 (1990) 131.

[6] S. L. Lukyanov and V. A. Fateev, Int. J. Mod. Phys. A7 (1992) 853; 1325.

[7] K. Gawedzki, Commun. Math. Phys. 139 (1991) 201.

[8] M. Chu, P. Goddard, I. Halliday, D. Olive, A. Schwimmer, Phys.Lett.B 266 (1991) 71.

[9] A. B. Zamolodchikov, unpublished.

[10] M. Wakimoto, Commun. Math. Phys. 104 (1986) 605.

[11] F. A. Smirnov, Dynamical symmetries of massive integrable models, I ; II, RIMS preprints $772 ; 838$.

[12] I. B. Frenkel and N. Yu. Reshetikhin, Commun. Math. Phys. 146 (1992) 1.

[13] O. Foda and T. Miwa, Int. J. Mod. Phys. A7 Suppl. 1A (1992) 279.

[14] B. Davies, O. Foda, M. Jimbo, T. Miwa and A.Nakayashiki, Diagonalization of the XXZ Hamiltonian by vertex operators, RIMS preprint 873 (1992).

[15] M. Jimbo, K. Miki, T. Miwa and A. Nakayashiki, Correlation Function of the XXZ model for $\Delta<-1$, RIMS preprint 877 (1992).

[16] A. N. Kirillov and F. A. Smirnov, Phys. Lett. 198B (1987) 506.

[17] F. A. Smirnov, Form factors in completely integrable models of quantum field theory, Advanced Series in Mathematical Physics 14, World Scientific (Singapure) 1992.

[18] V. G. Drinfeld, Quantum groups, Proc. ICM-86 (Berkeley), Vol.1 798 (1987).

[19] A. A. Belavin and V. G. Drinfeld, Funct. Anal. i ego Pril. 16 (1982) 1 (in Russian).

[20] N. Yu. Reshetikhin and M. A. Semenov-Tian-Shansky, Lett. Math. Phys. 19 (1990) 133.

[21] Jun'ichi Shriaishi, Free boson representation of $U_{q}(\widehat{s l(2)})$, Tokyo preprint, UT-617, september 92 .

[22] Akishsi Kato, Yas-Hiro Quano and Jun'ichi Shriaishi, Free boson representation of q-vertex operators and their correlation functions, Tokyo preprint UT-618, september 92. 